\title{
Laboratory Test Reference Range for Character Results Standard Unit
}

National Cancer Institute

\section{Source}

National Cancer Institute. Laboratory Test Reference Range for Character Results

Standard Unit. NCI Thesaurus. Code C83096.

The reference range of a laboratory test outcome, as reported in standard units. 